\title{
3,3'-Diindolylmethane mitigates lipopolysaccharide-induced acute kidney injury in mice by inhibiting NOX-mediated oxidative stress and the apoptosis of renal tubular epithelial cells
}

\author{
$\mathrm{JIN} \mathrm{HE}^{1^{*}}$, TAO HUANG $^{2 *}$ and LIN ZHAO ${ }^{2}$ \\ Departments of ${ }^{1}$ Nephrology and ${ }^{2}$ Emergency, The First Affiliated Hospital of Chongqing Medical University, \\ Chongqing 400016, P.R. China
}

Received October 11, 2018; Accepted March 29, 2019

DOI: $10.3892 / \mathrm{mmr} .2019 .10178$

\begin{abstract}
Diindolylmethane (DIM) is a naturally derived indole compound found in the Brassica family of vegetables. DIM has several beneficial effects, including anti-cancer, anti-inflammatory and anti-angiogenic functions. However, the effects of DIM on acute kidney injury (AKI) stimulated by lipopolysaccharide (LPS) are poorly studied. In this present study, male BALB/c mouse models of AKI were established using intraperitoneal injections of $10 \mathrm{mg} / \mathrm{kg}$ LPS. DIM $(40 \mathrm{mg} / \mathrm{kg})$ was administered intraperitoneally 24 and $2 \mathrm{~h}$ before LPS exposure. The results indicated that DIM significantly mitigated histopathological changes in the kidneys and improved the levels of blood urea nitrogen and serum creatinine. DIM also suppressed the LPS-induced production of reactive oxygen species and cell apoptosis. Furthermore, DIM treatment significantly decreased the expression of NADPH oxidase 2 (NOX2) and NOX4 in LPS-treated mice. Therefore, DIM may exert its renoprotective actions by inhibiting NOX-mediated oxidative stress and the apoptosis of renal tubular epithelial cells.
\end{abstract}

\section{Introduction}

Acute kidney injury (AKI), characterized by a sharp decline in renal function, is a severe complication with high morbidity and mortality rates and is commonly encountered in the intensive care unit (ICU) $(1,2)$. The Finnish Acute Kidney Injury study showed that AKI affects $40 \%$ of critically ill patients (3). AKI can be caused by various events, such as sepsis, cardiac surgery, liver or kidney transplantation,

Correspondence to: Dr Lin Zhao, Department of Emergency, The First Affiliated Hospital of Chongqing Medical University, 1 YiXueYuan Road, Yuzhong District, Chongqing 400016, P.R. China E-mail: zhaolincqmu@163.com

\section{${ }^{*}$ Contributed equally}

Key words: 3,3'-diindolylmethane, acute kidney injury, reactive oxygen species, apoptosis rheumatic fever, urinary tract obstruction, pharmacological toxins (4-6) and acute severe pancreatitis (7). Among these, endotoxic shock caused by lipopolysaccharide (LPS), which is the outer membrane component of gram-negative bacteria, is a common cause of AKI (8-10). Sepsis-associated AKI carries a particularly high mortality rate. One multicenter, international study involving an observational cohort of ICU patients demonstrated that the mortality rate of sepsis-related AKI patients was as high as $50 \%$ (2).

The mechanisms of sepsis-associated AKI are complex and not well understood. Although the mechanisms of sepsis-associated AKI are poorly understood, release of inflammatory factors, oxidative stress, apoptosis and microcirculatory dysfunction are believed to play an important role (11-13). So far, there are no effective drugs for the treatment of AKI. Therefore, novel and effective therapies to reduce the mortality of AKI are urgently needed.

3,3'-Diindolylmethane (DIM), a natural compound derived from the acid-catalyzed self-condensation of indole-3-carbinol, is abundant in cruciferous vegetables including kale and broccoli $(14,15)$. Studies have found that DIM can inhibit LPS-induced acute liver injury by regulating the expression of miRNAs $(14,16)$. Other studies have shown that DIM has a protective effect on the LPS-induced damage of cardiomyocytes and brain inflammation by reducing the release of pro-inflammatory mediators and negative regulation of the $\mathrm{NF}-\kappa \mathrm{B}$ signaling pathway $(17,18)$. In addition, DIM may also exert its organ protective function by mitigating oxidative stress and apoptosis (19).

However, the potentially protective characteristics of DIM have not yet been tested in LPS-triggered AKI. To address this, an experiment was designed to evaluate how DIM modifies disease progression in LPS-induced AKI.

\section{Materials and methods}

Animal protocols. Research protocols were reviewed and approved by the Experimental Animal Ethics Committee of Chongqing Medical University, while all the handling and care of animals were performed in strict compliance with the U.S. National Institute of Health Guide for the Care and Use of Laboratory Animals (1996 revision). Mice (male, aged 8 weeks 
old, body weight 22-25 g) were bred in a specific pathogen-free laboratory and allowed free access to food and tap water. The animal room was kept on a $12 \mathrm{~h}$ light/dark cycle at a constant temperature $\left(25^{\circ} \mathrm{C}\right)$ and relative humidity of $55 \pm 5 \%$ throughout the experimental period. Mice were divided into 4 groups of 10 mice (40 mice in total). The four groups comprised the control, LPS (10 mg/kg; Sigma Aldrich; Merck KGaA), DIM (40 mg/kg; MedChemExpress) and LPS + DIM (10 mg/kg LPS $+40 \mathrm{mg} / \mathrm{kg}$ DIM) groups. The choice of DIM concentration was based on a previous study (19) and incorporated similar DIM doses as administered in previous experiments $(14,19)$. An AKI murine model was produced by intraperitoneally injecting LPS (10 mg/kg in $200 \mu \mathrm{l}$ saline solution) and allowing $24 \mathrm{~h}$ for renal damage to develop. Mice were administered two intraperitoneal injections of DIM $(40 \mathrm{mg} / \mathrm{kg})$. The first and second doses were administered 24 and $2 \mathrm{~h}$ prior to the LPS inoculation, respectively. The animals were sacrificed after $24 \mathrm{~h}$ exposure to LPS and blood and kidney samples were harvested.

Histological examination. Tissues from the right kidney were first fixed in $4 \%$ paraformaldehyde $\left(4^{\circ} \mathrm{C}, 24 \mathrm{~h}\right)$ and embedded in paraffin. Sections of $4 \mu \mathrm{m}$ thickness were cut and processed and then stained with hematoxylin $(0.2 \%)$ and eosin (1\%) (H\&E) at room temperature for $30 \mathrm{sec}$ and $1 \mathrm{~min}$ respectively. Histological alterations in renal tissues and the degree of kidney injury were scored on a scale of $0-4$, as previously described (20), upon visualization by light microscopy (magnification, x400; Olympus Corporation).

Evaluation of renal function. Blood samples were extracted via the retro-orbital venous plexus and processed to extract the serum. In this present study, serum creatinine ( $\mathrm{SCr}$ ) and blood urea nitrogen (BUN) levels were employed as markers of renal function and were analyzed with an AutoAnalyzer (Roche Diagnostics $\mathrm{GmbH}$ ). SCr and BUN were quantified in accordance with the manufacturer's instructions.

TUNEL staining. Tissues of the right kidney were fixed in $4 \%$ paraformaldehyde, paraffin-embedded, and resected into $4 \mu \mathrm{m}$-thick sections according to the aforementioned procedure. TUNEL staining was conducted with a commercially available kit (Roche Diagnostics $\mathrm{GmbH}$ ) according to the manufacturer's instructions. Briefly, the dehydrated sections were treated with DNase-free proteinase $\mathrm{K}\left(37^{\circ} \mathrm{C}, 30 \mathrm{mins}\right)$, followed by $3 \% \mathrm{H}_{2} \mathrm{O}_{2}$ to quench endogenous peroxidase activity. Free $3^{\prime}-\mathrm{OH}$ termini were labeled with digoxigenin-dUTP for $1 \mathrm{~h}$ at $37^{\circ} \mathrm{C}$ using the TUNEL reaction mixture. Then, the sections were incubated with converter-POD at $37^{\circ} \mathrm{C}$ for $30 \mathrm{~min}$. DAB chromogenic reagent was employed to develop the stain and hematoxylin was used to stain the nucleus. Finally, the samples were cleared in xylene, mounted with neutral balsam and coverslipped. Under optical microscopy (magnification, $\mathrm{x} 400$; Olympus Corporation), the number of TUNEL-positive cells in 400 histological fields were counted per kidney section.

Fluorescence microscopy analysis for kidney reactive oxygen species (ROS). ROS generated in the kidney were detected using dihydroethidium (DHE; Beyotime Institute of Biotechnology). The left kidney tissues were stored in liquid nitrogen before preparation into frozen sections. The kidney sections were washed three times with ice-cold PBS, treated with DHE $\left(10^{-5} \mathrm{M}\right.$, final concentration) and incubated for $30 \mathrm{~min}$ at $37^{\circ} \mathrm{C}$. After treatment, sections were again washed three times with ice-cold PBS. The excitation (480 $\mathrm{nm}$ ) and emission $(590 \mathrm{~nm})$ wavelengths for these experiments were set by fluorescent microscopy. All mean fluorescence values were analyzed by comparing each group using the ZEN 2012 software (Carl Zeiss AG, Germany).

Measurement of kidney lipid peroxidation and glutathione $(G S H)$ content. The malondialdehyde (MDA) level in kidney homogenates was analyzed by measuring the level of trimethine, a reaction product of MDA and thiobarbituric acid, spectrophotometrically at a wavelength of $535 \mathrm{~nm}$. The reduced GSH level in the kidney homogenates was determined using 5,5'-dithiobis-2-nitrobenoicacid (DTNB) at a wavelength of $412 \mathrm{~nm}$. Thereafter, oxidized GSH (GSSG) is reduced to GSH by GSH reductase, total GSH (T-GSH) is measured by the same method. The level of GSSG can be calculated by subtracting GSH from T-GSH.

Western blot analysis. A protein extraction kit (RIPA Lysis Buffer, Beyotime Institute of Biotechnology) was used to isolate proteins from the left renal tissue samples. The bicinchoninic acid method was employed to quantify protein concentrations. Each protein sample of $20 \mu \mathrm{g}$ was separated via $10 \%$ SDS-PAGE gel electrophoresis and transferred to a PVDF membrane (EMD Millipore) and successively incubated with $5 \%$ non-fat milk at room temperature for $1.5 \mathrm{~h}$. PVDF membranes were allowed to incubate overnight at $4{ }^{\circ} \mathrm{C}$ with the indicated primary antibodies, including caspase-3 (1:500; cat. no. wl01927; Wanleibio Co., Ltd.), cleaved caspase-3 (1:500; cat. no. wl01992; Wanleibio Co., Ltd.), poly (ADP-ribose) polymerase (PARP; 1:500; cat. no. wl0326; Wanleibio Co., Ltd.), cleaved PARP (1:500; cat. no. w101932; Wanleibio co., Ltd.), NADPH oxidase-1, $-2,-3$ and -4 (NOX1, NOX2, NOX3 and NOX4, respectively; 1:500; sc-518023, sc-17844, sc-7662 and sc-20781, respectively; Santa Cruz Biotechnology, Inc.,USA), and $\beta$-actin (1:1,000; cat. no. A0208; Beyotime Institute of Biotechnology). The following day, HRP labeled goat anti-rabbit IgG $(\mathrm{H}+\mathrm{L})(1: 3,000$; Beyotime Institute of Biotechnology) was added and further incubated at room temperature for $1 \mathrm{~h}$. Immunoreactive bands were visualized using an ECL (cat. no. WLA003a, Wanleibio co., Ltd.) system and quantified with using Image $\mathbf{J}$ software (version 1.8.0).

Statistical analysis. All experiments were conducted at least three times. All data are presented as the mean \pm standard error of the mean. All statistical analyses were performed using SPSS 17.0 statistical software (SPSS, Inc.). Statistical significance of multiple groups was determined by one-way ANOVA, followed by the least significant difference for multiple comparisons test. $\mathrm{P}<0.05$ was considered to indicate a statistically significant difference.

\section{Results}

DIM mitigates histopathological changes and restores renal function in LPS-induced AKI mouse models. To determine how DIM affected the progression of LPS-mediated AKI, the kidneys were examined for histopathological changes. 


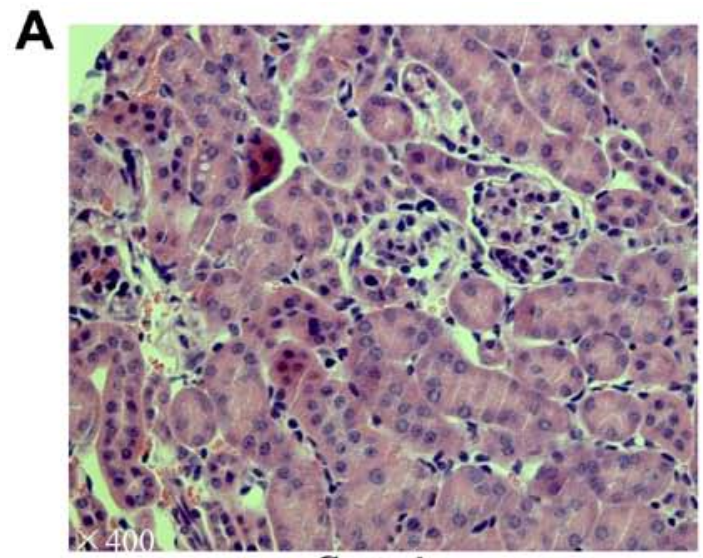

Control

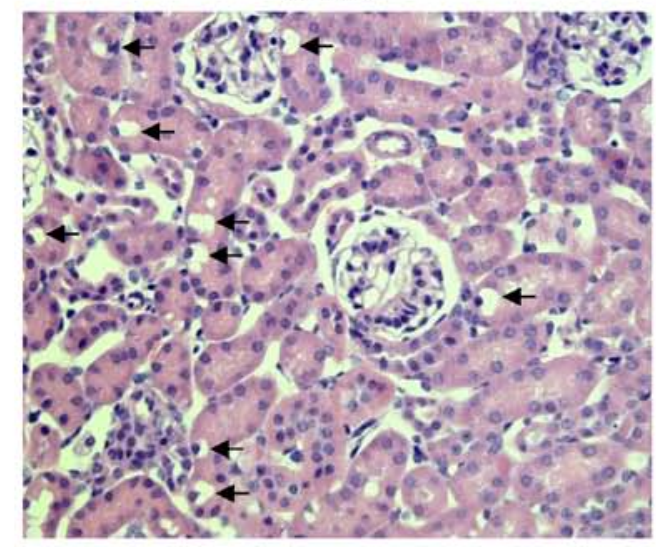

LPS
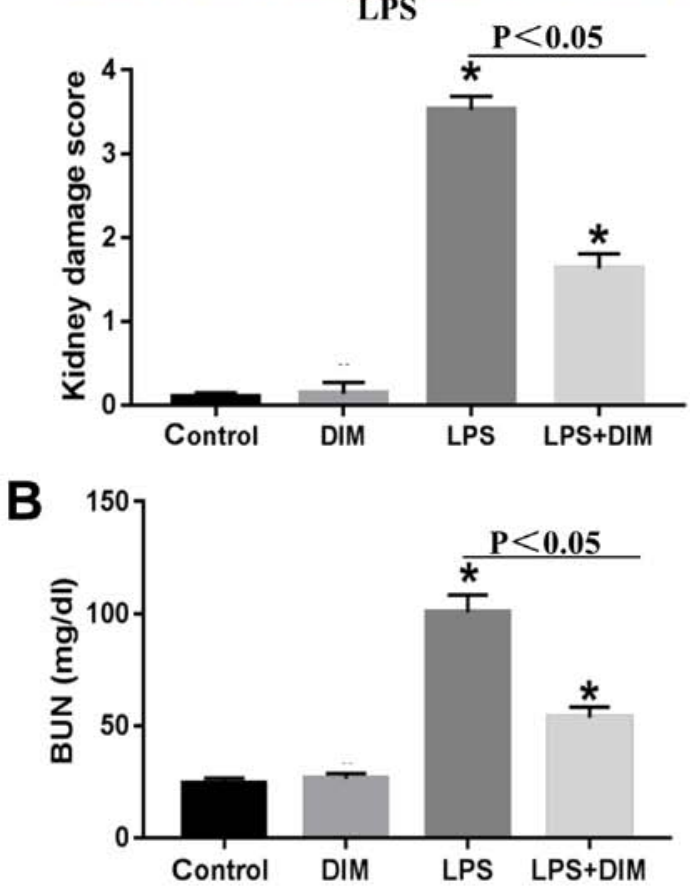

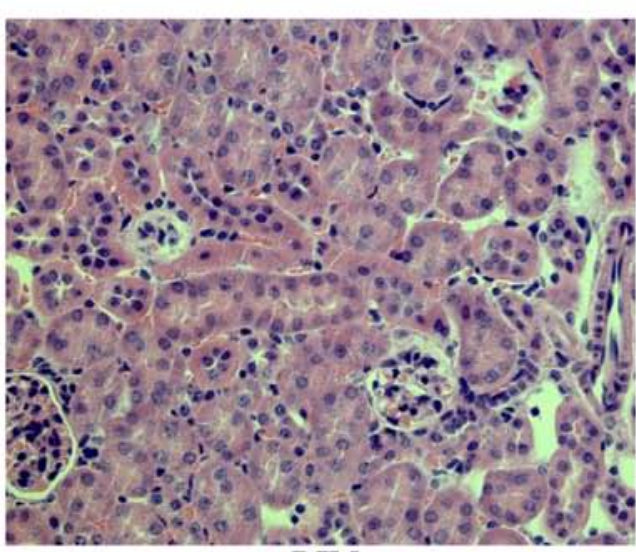

DIM

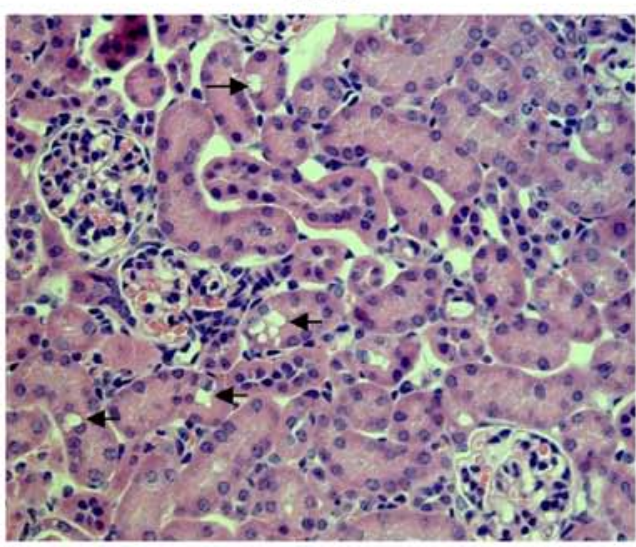

LPS+DIM

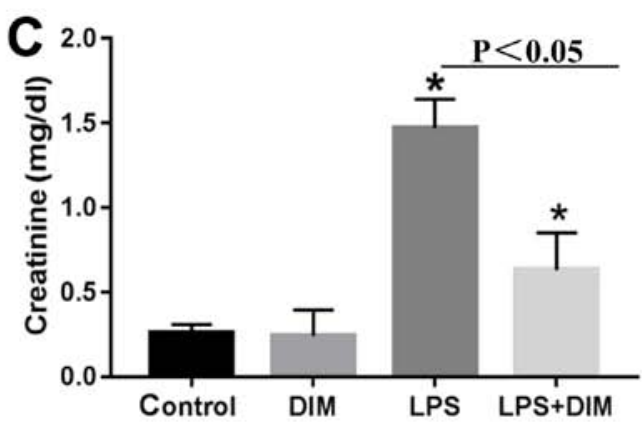

Figure 1. DIM mitigates histopathological changes and restores renal function in an LPS-induced acute kidney injury mouse model. (A) Mice were exposed to LPS (10 mg/kg), DIM (40 mg/kg), or LPS + DIM (10 mg + $40 \mathrm{mg})$ for $24 \mathrm{~h}$; histological changes in kidneys were observed by hematoxylin and eosin staining. (B) BUN levels were determined after the mice were exposed to LPS, DIM or LPS + DIM for 24 h. (C) Blood creatinine levels were determined after the mice were exposed to LPS, DIM or LPS + DIM for $24 \mathrm{~h}$. The arrows indicate damaged tubular epithelial cells. The results are presented as the mean \pm SEM. ${ }^{*}<<0.05$ vs. control. DIM, 3,3'-diindolylmethane; LPS, lipopolysaccharide; BUN, blood urea nitrogen.

Mice that were exposed to LPS exhibited several indicators of AKI, including destroyed renal tubules, aberrant renal tubular epithelial cells, interstitial edema in renal epithelial cells and renal tubule dilation (Fig. 1A). H\&E staining showed that the epithelial cell structure and the glomerular membrane were normal in the control and DIM groups. AKI-related kidney lesions appeared to be attenuated in AKI mouse models treated with DIM. 
A

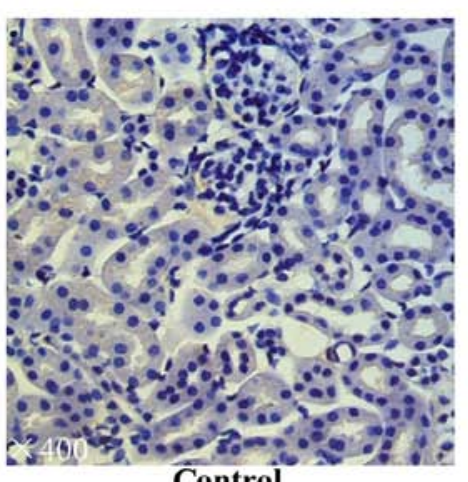

Control

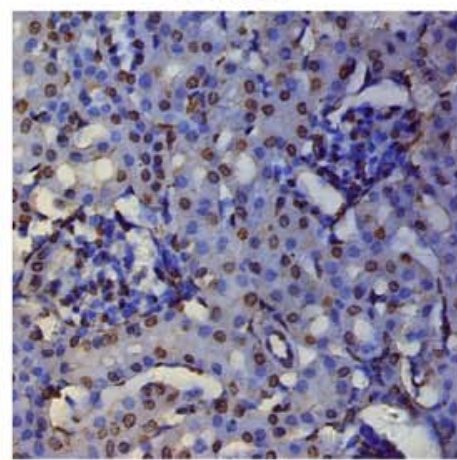

LPS

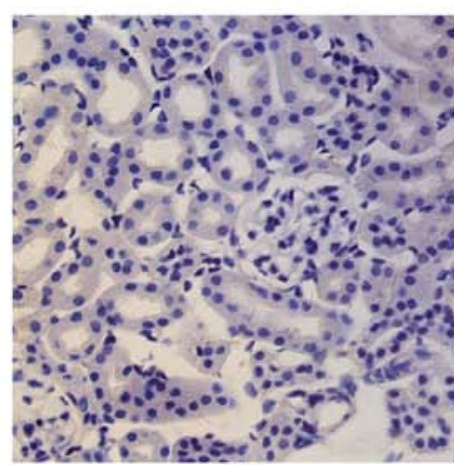

DIM

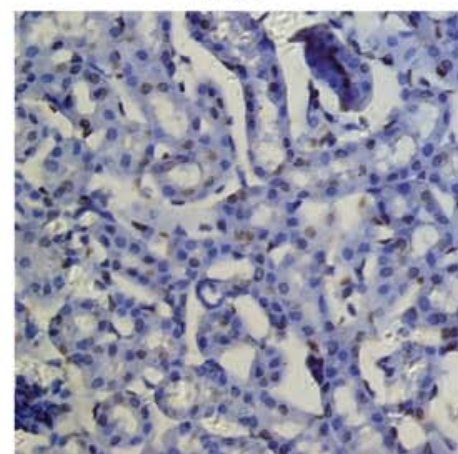

LPS+DIM

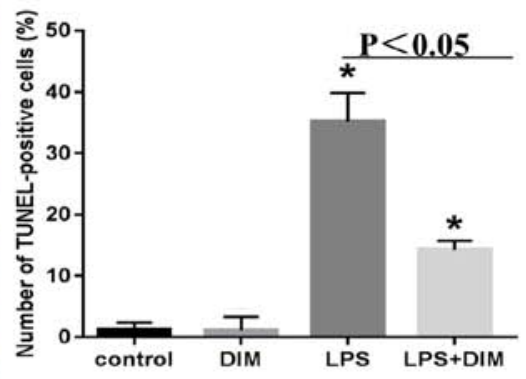

B

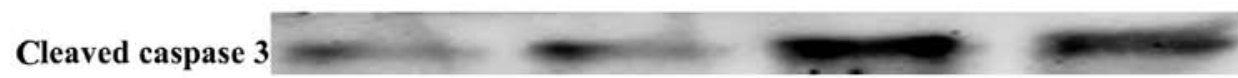

Caspase 3

Cleaved PARP
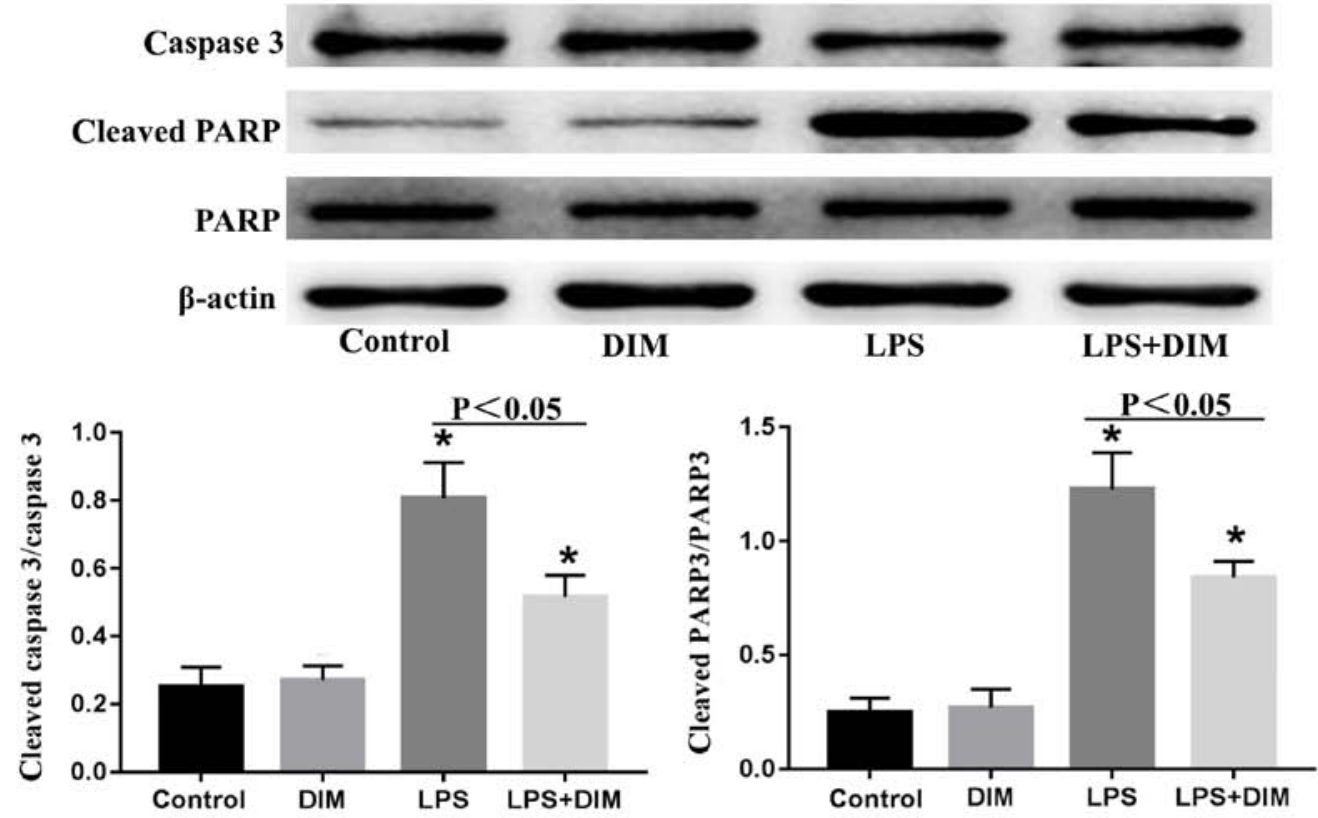

Figure 2. DIM treatment inhibits the apoptosis of renal tubular epithelial cells in mice with LPS-triggered acute kidney injury. Mice were exposed to LPS $(10 \mathrm{mg} / \mathrm{kg})$, DIM $(40 \mathrm{mg} / \mathrm{kg})$ or LPS + DIM $(10 \mathrm{mg}+40 \mathrm{mg})$ for $24 \mathrm{~h}$ after which (A) the apoptosis of renal tubular epithelial cells was determined by TUNEL staining and (B) the expression levels of cleaved caspase-3 and cleaved PARP were determined by western blot analysis. "P<0.05 vs. control. DIM, 3,3'-diindolylmethane; LPS, lipopolysaccharide; PARP, poly (ADP-ribose) polymerase.

To further confirm the results from the H\&E staining, changes in $\mathrm{SCr}$ and BUN levels were also examined. As shown in Fig. $1 \mathrm{~B}$ and $\mathrm{C}$, DIM significantly improved the renal function of AKI mice.

DIM treatment inhibits the apoptosis of renal tubular epithelial cells in mice with LPS-triggered AKI. As the apoptosis of renal tubular epithelial cells is an important mechanism leading to AKI, the effects of DIM on LPS-induced tubular epithelial cell apoptosis were examined.

As shown in Fig. 2A, numerous TUNEL-positive renal tubular epithelial cells were observed in the kidney tissues of the LPS-induced AKI mouse models. However, fewer TUNEL-positive tubular epithelial cells were observed in 


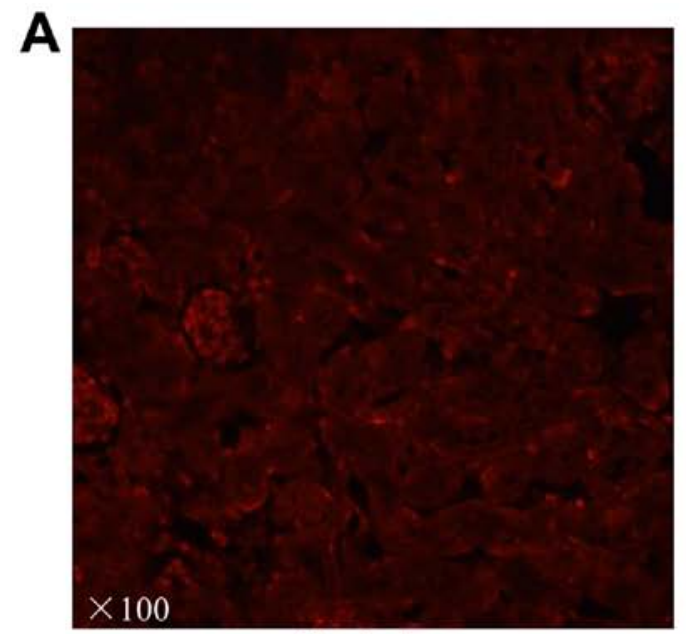

Control

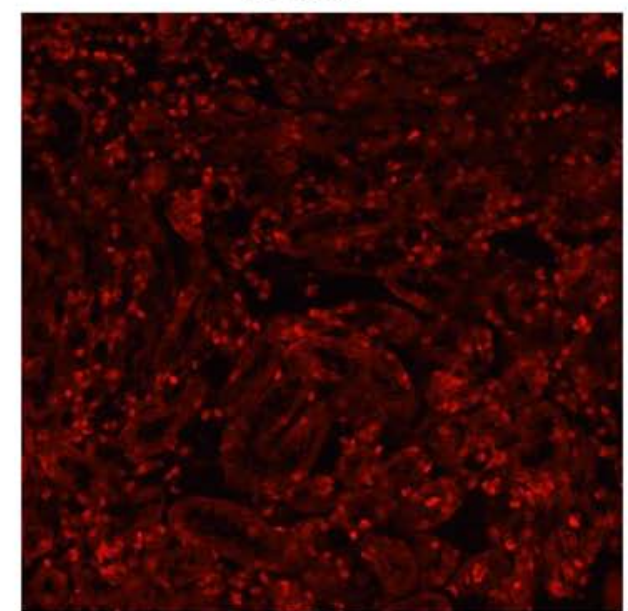

LPS

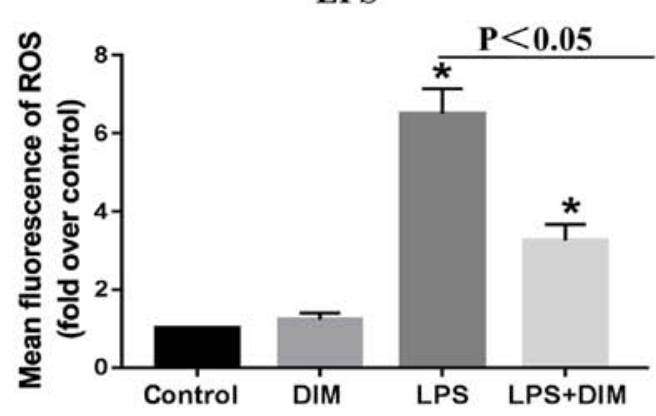

B

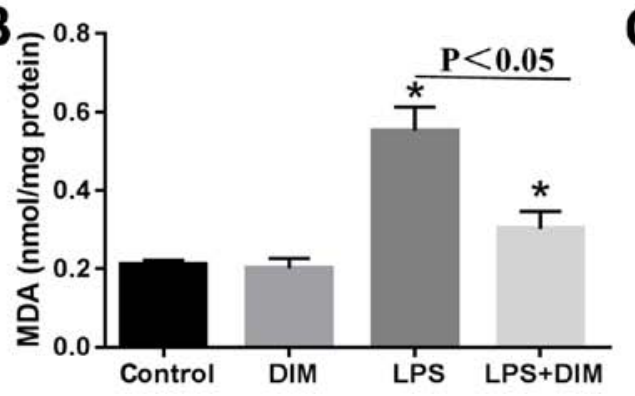

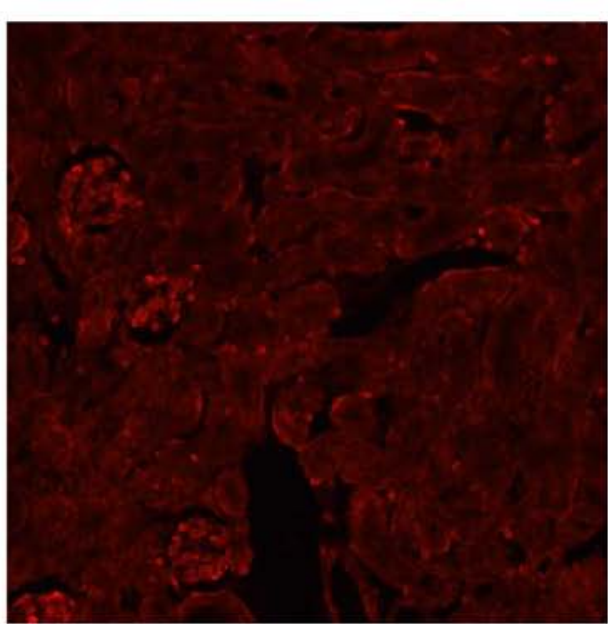

DIM

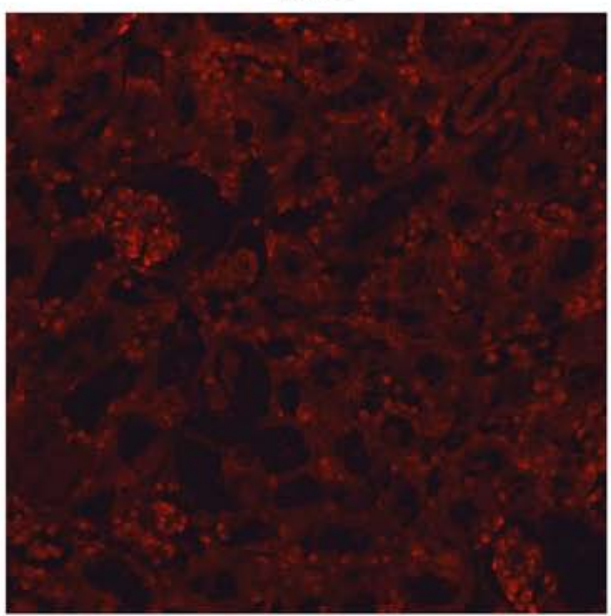

LPS+DIM

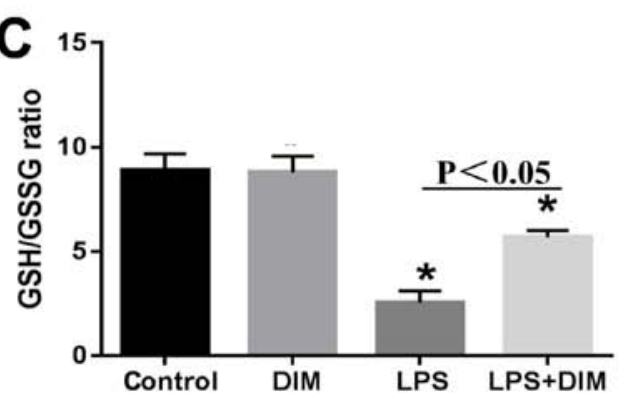

Figure 3. DIM treatment alleviates oxidative stress in mice with LPS-triggered acute kidney injury. Mice were exposed to LPS (10 mg/kg), DIM (40 mg/kg) or LPS + DIM (10 mg + $40 \mathrm{mg}$ ) for $24 \mathrm{~h}$ after which (A) ROS production was observed by dihydroethidium, (B) MDA levels in the kidney tissues were determined and (C) the GSH/GSSG ratio in the kidney tissues was calculated. "P<0.05 vs. control. DIM, 3,3'-diindolylmethane; LPS, lipopolysaccharide, ROS, reactive oxygen species; MDA, malondialdehyde; GSH/GSSG, glutathione/oxidized glutathione.

the kidney tissues of the DIM-treated AKI mice. Consistent with this, western blot analysis revealed that the expression levels of apoptotic markers, including cleaved-caspase-3 and cleaved-PARP, were significantly increased in the LPS-induced AKI mouse model and were attenuated by DIM pre-treatment (Fig. 2B). 

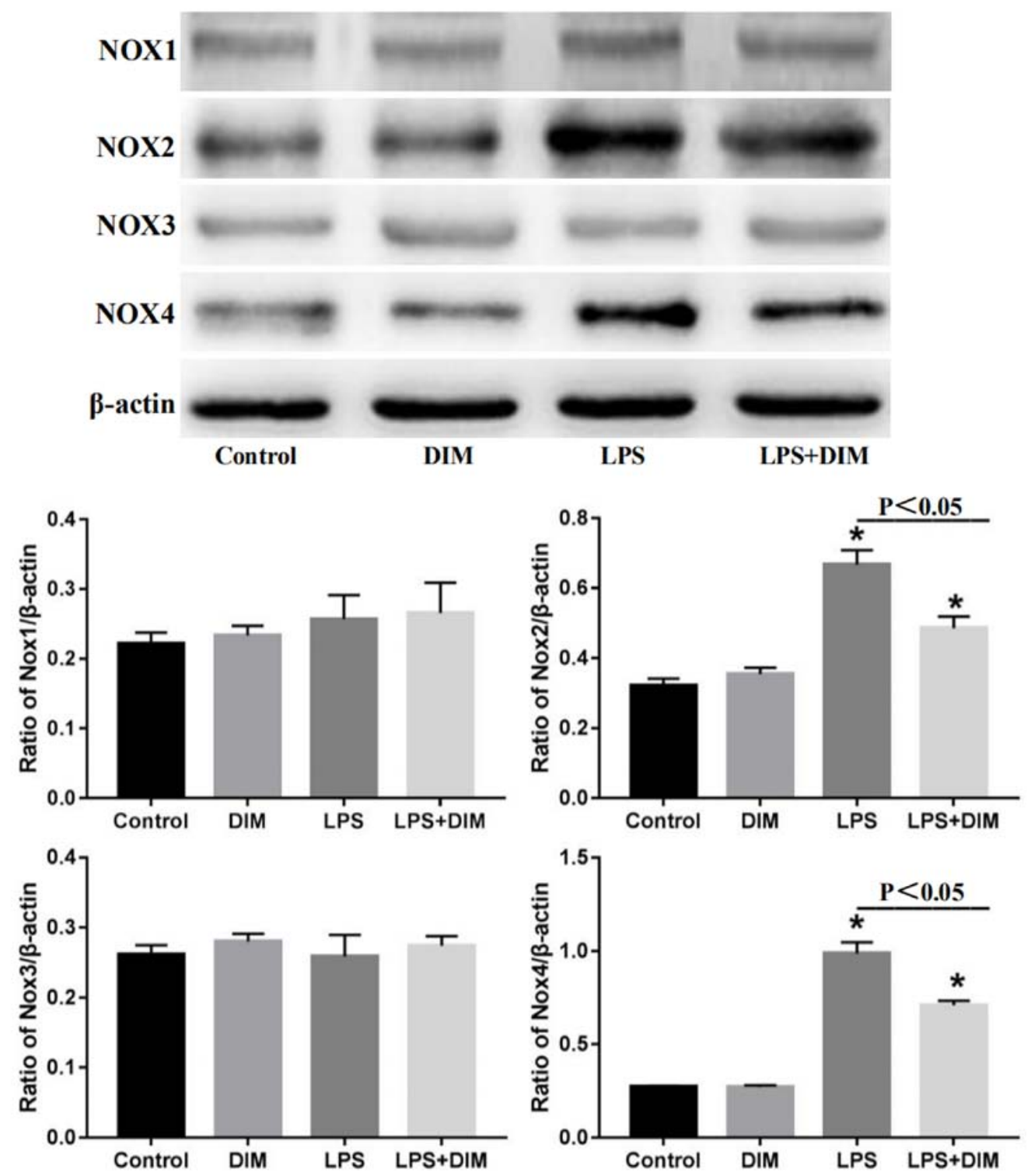

Figure 4. DIM treatment suppresses the expression of NOX2 and NOX4 in mice with LPS-triggered acute kidney injury. Mice were exposed to LPS (10 mg/kg), DIM $(40 \mathrm{mg} / \mathrm{kg})$ or LPS + DIM (10 mg + $40 \mathrm{mg})$ for $24 \mathrm{~h}$ after which the expression levels of NOX1, NOX2, NOX3 and NOX4 were determined by western blot analysis. "P<0.05 vs. control. DIM, 3,3'-diindolylmethane; NOX, NADPH oxidase; LPS, lipopolysaccharide.

DIM treatment alleviates oxidative stress in mice with $L P S$-triggered AKI. To further elucidate the mechanisms allowing DIM to mediate its anti-apoptotic effects on LPS-induced cell apoptosis, alterations in oxidative stress were investigated. Mice that received LPS had elevated levels of ROS and MDA and a decreased GSH/GSSG ratio in comparison with the control and DIM groups (Fig. 3). The alterations in oxidative stress indexes significantly improved when treated with DIM.

DIM treatment suppresses the expression of NOX2 and NOX4 in mice with LPS-triggered AKI. As the NOX enzymes are major source of ROS in the kidneys, the effect of DIM on the expression of NOX family members was tested. As shown in Fig. 4, LPS had no effects on the expression of NOX1 and NOX3, but significantly promoted the expression of NOX2 and NOX4. Nevertheless, DIM effectively blocked the LPS-induced expression of NOX2 and NOX4.

\section{Discussion}

AKI, which often arises in sepsis cases, is a common problem in critical patients and carries high morbidity and mortality rates $(2,21)$. However, effective medical treatments for AKI are not commonly available. In the present study, the effects of DIM on LPS-induced AKI in mice was tested. DIM conferred protection against AKI induced by LPS, as demonstrated by the improvements in kidney function and the attenuation of the kidney damage score. These effects may be achieved by inhibiting the NOX-mediated oxidative stress and programmed cell death in renal tubular epithelial cells.

Apoptosis is an important pathological mechanism leading to AKI $(13,22-25)$. As expected, the LPS-induced AKI mouse model showed increased apoptosis in renal tubular epithelial cells, as determined by the elevated expression levels of cleaved caspase- 3 and cleaved PARP measured by western blot analysis. Consistent with the effects of DIM on renal 
function in the AKI mouse model, DIM could decrease the levels of both cleaved caspase-3 and cleaved PARP in kidneys stimulated with LPS. TUNEL staining also showed that DIM could alleviate the level of apoptosis in LPS-induced tubular epithelial cells. Thus, DIM may mitigate LPS-induced AKI by reducing the level of apoptosis in renal tubular epithelial cells.

ROS play an important role in apoptosis induction under both physiological and pathological conditions (26). LPS is a strong inducer of ROS production, and the excessive production of ROS is closely related to the apoptosis of renal tubular cells by causing the release of apoptotic factors, including cytochrome $\mathrm{C}$, and by destroying the permeability of the mitochondrial membrane. Aside from the actions on mitochondria, ROS may also activate sphingomyelinase-generating ceramide, an intracellular mediator of apoptosis in granulocytes (25-28). In the present study, mice challenged with LPS showed a significant increase in ROS and MDA (lipid peroxidation) levels coinciding with a rapid decrease in the GSH/GSSG ratio. Reduced GSH plays an important role as a free radical scavenger to counteract the deleterious effects of ROS (29). These changes were all attenuated by DIM. Thus, DIM may protect against LPS-induced apoptosis by reducing oxidative stress.

ROS are primarily generated in the mitochondria by NOX enzymes, cyclooxygenase and xanthine oxidase. The NOX family is the main source of ROS in the kidneys (30-32). To determine the mechanism by which DIM reduces ROS, the effect of DIM on the expression of NOX enzymes (NOX1-NOX4) was investigated. The data revealed that LPS promoted ROS production primarily through NOX2 and NOX4. The expression of NOX2 and NOX4 was decreased in the presence of DIM. Taken together, the data presented in this present study support the hypothesis that DIM suppresses LPS-triggered oxidative stress by inhibiting the NOX2 and NOX4 pathways.

A total of three major findings have been revealed in this present study. First, DIM significantly improved renal function as demonstrated by the lowering of SCr and BUN levels, as well as the attenuation of pathological kidney damage in the LPS-stimulated AKI mouse model. Secondly, DIM suppressed the pro-apoptotic effects of LPS. Finally, DIM may confer its protective benefits by inhibiting NOX/ROS signaling.

In conclusion, DIM is a potential therapeutic agent for use in AKI triggered by LPS. The possible mechanism through which DIM attenuates renal damage in the LPS-induced AKI mouse model is via the inhibition of NOX-mediated oxidative stress and programmed cell death of renal tubular epithelial cells. This present study focused on an animal model of AKI and was not sufficient to describe how DIM acts on AKI through the regulation of NOX-mediated oxidative stress and programmed cell death of renal tubular epithelial cells. Therefore, further studies using cells are required to elucidate the specific mechanism of DIM regulation.

\section{Acknowledgements}

Not applicable.

\section{Funding}

No funding was received.

\section{Availability of data and materials}

The datasets generated and analyzed during the present study are available from the corresponding author on reasonable request.

\section{Authors' contributions}

$\mathrm{JH}$ and $\mathrm{TH}$ performed the experiments, data analysis and manuscript writing. LZ designed the experiments, participated in the generation of ideas and data interpretation and was responsible for the overall direction of this work. All authors read and approved the final manuscript.

\section{Ethics approval and consent to participate}

The study protocol was reviewed and approved by the Animal Care and Use Committee of Chongqing Medical University.

\section{Patient consent for publication}

Not applicable.

\section{Competing interests}

The authors declare that they have no competing interests.

\section{References}

1. Hoste EA and Kellum JA: Acute kidney injury: Epidemiology and diagnostic criteria. Curr Opin Crit Care 12: 531-537, 2006.

2. Uchino S, Kellum JA, Bellomo R, Doig GS, Morimatsu H, Morgera S, Schetz M, Tan I, Bouman C, Macedo E, et al: Acute renal failure in critically ill patients: A multinational, multicenter study. JAMA 294: 813-818, 2005.

3. Nisula S, Kaukonen KM, Vaara ST, Korhonen AM, Poukkanen M, Karlsson S, Haapio M, Inkinen O, Parviainen I, Suojaranta-Ylinen R, et al: Incidence, risk factors and 90-day mortality of patients with acute kidney injury in Finnish intensive care units: The FINNAKI study. Intensive Care Med 39: 420-428, 2013.

4. Kellum JA, Lameire N, Aspelin P, Barsoum RS, Burdmann EA, Goldstein SL, Herzog CA, Joannidis M, Kribben A, Levey AS, et al: Kidney disease: Improving global outcomes (KDIGO) acute kidney injury work group. KDIGO clinical practice guideline for acute kidney injury. Kidney Int Suppl 2: $1-138,2012$.

5. Liang R, Zhao Q, Jian G, Cheng D, Wang N, Zhang G and Wang F: Tanshinone IIA attenuates contrast-induced nephropathy via Nrf2 activation in rats Cell Physiol Biochem 46: 2616-2623, 2018.

6. Wu R, Kong Y, Yin J, Liang R, Lu Z, Wang N, Zhao Q, Zhou Y, Yan C, Wang F and Liang M: Antithrombin III is a novel predictor for contrast induced nephropathy after coronary angiography. Kidney Blood Press Res 43: 170-180, 2018.

7. Kong Y, Yin J, Cheng D, Lu Z, Wang N, Wang F and Liang M: Antithrombin III attenuates AKI following acute severe pancreatitis. Shock 49: 572-579, 2018.

8. Mårtensson J and Bellomo R: Pathophysiology of septic acute kidney injury. Contrib Nephrol 187: 36-46, 2016.

9. Skube SJ, Katz SA, Chipman JG and Tignanelli CJ: Acute kidney injury and sepsis. Surg Infect (Larchmt) 19: 216-224, 2018.

10. Zarbock A, Gomez H and Kellum JA: Sepsis-induced acute kidney injury revisited: Pathophysiology, prevention and future therapies. Curr Opin Crit Care 20: 588-595, 2014.

11. Gomez H, Ince C, De Backer D, Pickkers P, Payen D, Hotchkiss J and Kellum JA: A unified theory of sepsis-induced acute kidney injury: Inflammation, microcirculatory dysfunction, bioenergetics, and the tubular cell adaptation to injury. Shock 41: 3-11, 2014. 
12. Jacobs R, Honore PM, Joannes-Boyau O, Boer W, De Regt J, De Waele E, Collin V and Spapen HD: Septic acute kidney injury: The culprit is inflammatory apoptosis rather than ischemic necrosis. Blood Purif 32: 262-265, 2011.

13. Wan L, Bagshaw SM, Langenberg C, Saotome T, May C and Bellomo R: Pathophysiology of septic acute kidney injury: What do we really know? Crit Care Med 36 (4 Suppl): S198-S203, 2008.

14. Tomar S, Nagarkatti M and Nagarkatti PS: 3,3'-Diindolylmethane attenuates LPS-mediated acute liver failure by regulating miRNAs to target IRAK4 and suppress Toll-like receptor signalling. Br J Pharmacol 172: 2133-2147, 2015.

15. Cho HJ, Seon MR, Lee YM, Kim J, Kim JK, Kim SG and Park JH: 3,3'-Diindolylmethane suppresses the inflammatory response to lipopolysaccharide in murine macrophages. J Nutr 138: 17-23, 2008.

16. Busbee PB, Nagarkatti $M$ and Nagarkatti PS: Natural indoles, indole-3-carbinol (I3C) and 3,3'-diindolylmethane (DIM), attenuate staphylococcal enterotoxin B-mediated liver injury by downregulating miR-31 expression and promoting caspase-2-mediated apoptosis. PLoS One 10: e0118506, 2015.

17. Li J, Wu Q, Deng W and Tang Q: GW26-e4573 Anti-inflammatory effect of 3,3'-Diindolylmethane on LPS-induced inflammatory injury in neonatal rat cardiac myocytes via suppressing TLR-4/MAPKs signaling pathways. J Am College Cardiol 66: C59, 2015.

18. Kim HW, Kim J, Kim J, Lee S, Choi BR, Han JS, Lee KW and Lee HJ: 3,3'-Diindolylmethane inhibits lipopolysaccharide-induced microglial hyperactivation and attenuates brain inflammation. Toxicol Sci 137: 158-167, 2014.

19. Hajra S, Basu A, Singha Roy S, Patra AR and Bhattacharya S: Attenuation of doxorubicin-induced cardiotoxicity and genotoxicity by an indole-based natural compound 3,3'-diindolylmethane (DIM) through activation of Nrf2/ARE signaling pathways and inhibiting apoptosis. Free Radic Res 51: 812-827, 2017.

20. Tilyek A, Chai C, Hou X, Zhou B, Zhang C, Cao Z and Yu B: The protective effects of Ribes diacanthum Pall on cisplatin-induced nephrotoxicity in mice. J Ethnopharmacol 178: 297-306, 2016.

21. Zarjou A and Agarwal A: Sepsis and acute kidney injury. J Am Soc Nephrol 22: 999-1006, 2011.

22. Cantaluppi V, Quercia AD, Dellepiane S, Figliolini F, Medica D and De Lena $M$ : New mechanisms and recent insights in the pathogenesis of acute kidney injury (AKI). G Ital Nefrol 29 . 535-547, 2012 (In Italian).
23. Langenberg C, Bagshaw SM, May CN and Bellomo R: The histopathology of septic acute kidney injury: A systematic review. Crit Care 12: R38, 2008

24. Lerolle N, Nochy D, Guérot E, Bruneval P, Fagon JY, Diehl JL and Hill G: Histopathology of septic shock induced acute kidney injury: Apoptosis and leukocytic infiltration. Intensive Care Med 36: 471-478, 2010.

25. Bae EH, Kim IJ, Choi HS, Kim HY, Kim CS, Ma SK, Kim IS and Kim SW: Tumor necrosis factor $\alpha$-converting enzyme inhibitor attenuates lipopolysaccharide-induced reactive oxygen species and mitogen-activated protein kinase expression in human renal proximal tubule epithelial cells. Korean J Physiol Pharmacol 22: 135-143, 2018.

26. Simon HU, Haj-Yehia A and Levi-Schaffer F: Role of reactive oxygen species (ROS) in apoptosis induction. Apoptosis 5: 415-418, 2000

27. Nordberg J and Arnér ES: Reactive oxygen species, antioxidants, and the mammalian thioredoxin system. Free Radic Biol Med 31: 1287-1312, 2001

28. Kroemer G and Reed JC: Mitochondrial control of cell death. Nat Med 6: 513-519, 2000.

29. Kim J, Kim HY and Lee SM: Protective effects of geniposide and genipin against hepatic ischemia/reperfusion injury in mice. Biomol Ther (Seoul) 21: 132-137, 2013.

30. Shiose A, Kuroda J, Tsuruya K, Hirai M, Hirakata H, Naito S, Hattori M, Sakaki Y and Sumimoto H: A novel superoxide-producing NAD(P)H oxidase in kidney. J Biol Chem 276: $1417-1423,2001$

31. Gao L, Wu WF, Dong L, Ren GL, Li HD, Yang Q, Li XF, Xu T, Li Z, Wu BM, et al: Protocatechuic aldehyde attenuates cisplatin-induced acute kidney injury by suppressing nox-mediated oxidative stress and renal inflammation. Front Pharmacol 7: 479, 2016.

32. Zhang J, Wang X, Vikash V, Ye Q, Wu D, Liu Y and Dong W: ROS and ROS-mediated cellular signaling. Oxid Med Cell Longev 2016: 4350965, 2016.

This work is licensed under a Creative Commons Attribution-NonCommercial-NoDerivatives 4.0 International (CC BY-NC-ND 4.0) License. 